\title{
Synthesis, Characterization, and Evaluation of a Novel Amphiphilic Polymer RGD-PEG-Chol for Target Drug Delivery System
}

\author{
Shi Zeng, ${ }^{1}$ Fengbo Wu, ${ }^{1}$ Bo Li, ${ }^{1}$ Xiangrong Song, ${ }^{1}$ Yu Zheng, ${ }^{1}$ Gu He, \\ Cheng Peng, ${ }^{2}$ and Wei Huang ${ }^{2}$ \\ ${ }^{1}$ State Key Laboratory of Biotherapy and Department of Pharmacy, West China Hospital, Sichuan University, \\ No. 37 Guoxue Alley, Chengdu, Sichuan 610041, China \\ ${ }^{2}$ State Key Laboratory Breeding Base of Systematic research, Development and Utilization of Chinese Medicine, \\ Chengdu University of Traditional Chinese Medicine, Chengdu 611137, China
}

Correspondence should be addressed to Gu He; heguscu@163.com

Received 26 August 2013; Accepted 24 October 2013; Published 21 January 2014

Academic Editors: G. Lamberti and S. Mao

Copyright (C) 2014 Shi Zeng et al. This is an open access article distributed under the Creative Commons Attribution License, which permits unrestricted use, distribution, and reproduction in any medium, provided the original work is properly cited.

\begin{abstract}
An amphiphilic polymer RGD-PEG-Chol which can be produced in large scale at a very low cost has been synthesized successfully. The synthesized intermediates and final products were characterized and confirmed by ${ }^{1} \mathrm{H}$ nuclear magnetic resonance spectrum $\left({ }^{1} \mathrm{H}\right.$ NMR) and Fourier transform infrared spectrum (FT-IR). The paclitaxel- (PTX-) loaded liposomes based on RGD-PEG-Chol were then prepared by film formation method. The liposomes had a size within $100 \mathrm{~nm}$ and significantly enhanced the cytotoxicity of paclitaxel to B16F10 cell as demonstrated by MTT test $\left(\mathrm{IC}_{50}=0.079 \mu \mathrm{g} / \mathrm{mL}\right.$ of RGD-modified PTX-loaded liposomes compared to $9.57 \mu \mathrm{g} / \mathrm{mL}$ of free PTX). Flow cytometry analysis revealed that the cellular uptake of coumarin encapsulated in the RGDPEG-Chol modified liposome was increased for HUVEC cells. This work provides a reasonable, facile, and economic approach to prepare peptide-modified liposome materials with controllable performances and the obtained linear RGD-modified PTX-loaded liposomes might be attractive as a drug delivery system.
\end{abstract}

\section{Introduction}

During the last decade, many reports have been focused on developing colloidal carrier systems for a variety of anticancer drugs to achieve targeted drug delivery to tumor sites and improve their intracellular uptake. Amphiphilic polymers have received increasing attention because of their desired chemical, interfacial, mechanical, and biological functions, which make them a suitable platform for sustained drug release [1-4] and a promising carrier for therapeutic agents (especially poorly soluble drugs) [5]. Meanwhile, the development of improved synthetic methodologies has greatly supported the growth of this field $[6,7]$.

Liposomal delivery has been extensively investigated in cancer treatment. To avoid rapid clearance of liposomes from the circulation by the reticuloendothelial system, sterically stabilized liposomes (SSL) have been developed. The moieties of poly(ethylene glycol)s (PEGs) as membrane active agents are commonly used within modern pharmaceutical chemistry, because it could extend in "mushroom" or "brush" chain conformation from the membrane plane towards the water solution and form thick hydrophilic layers at the membrane surface [8]. The functionalization of PEG allows subsequent attachment of biologically active or even celltargeting molecules to prepare sterically stabilized liposomes which retain long survival time in circulation and target recognition [9]. In our work, PEG2000 was chosen since it can covalently attach to the lipid head group, undergo steric exclusion from the liposome surface, and play an important role in thermodynamic stability of liposomes by dehydrating the lipid bilayer [10-14].

Although differential accumulation of liposomes carrying drugs in tumor sites relative to normal tissues can be achieved 
by the effect of enhanced permeability and retention (EPR), it is still necessary to improve inefficient intracellular uptake of the entrapped anticancer drugs by the tumor cells by active targeting strategy [15-17]. Integrin is a family of cell surface receptors responsible for anchoring cells to the extracellular matrix (ECM) and they are overexpressed in the melanoma tumor cells and tumor endothelial cells [18]. The RGD (Arg-Gly-Asp) sequence can bind to several integrins such as integrin $a_{\mathrm{v}} \beta_{3}$ and $a_{5} \beta_{1}$ [19]. In this paper, we used a linear RGD peptide to construct an amphiphilic polymer cholesterol-poly(ethylene glycol)-RGD and used it to establish a RGD-modified liposomal drug delivery system for paclitaxel (PTX), a natural antimicrotubule agent for treating a variety of solid tumors, such as ovarian cancer, breast cancer, and lung cancer [20, 21].

RGD-modified polymers have been investigated extensively and there are also reports about Lipid-PEG-RGD polymers which are similar to our work $[22,23]$. These works are very extraordinary, impressive and laid a solid foundation for our study. Compared with their outstanding work, however, we also have our own advantages. In our work, we choose cholesterol as biomembrane anchor instead of artificial lipids such as 1,2-distearoyl-sn-glycero-3-phosphoethanolamine (DSPE) because of the fact that cholesterol gives liposomal systems similar mechanical and biological functions compared to DSPE [24-27] and that the cost of cholesterol is over 100 times lower than that of DSPE, which means it can be easily synthesized in large amounts on an industrial scale. In our work, we choose ordinary PEG instead of FmocPEG-CO ${ }_{2}$ NHS, which had been reported, since ordinary PEG is far more cost-efficient. Also the synthesis route we designed is more simple and facile, and there is no need to use purification methods such as HPLC which cost more money and time. In this way, our method leads to higher product rate and the possibility of producing in large scale.

The aim of this paper was to synthesize cholesterolpoly(ethylene glycol)-RGD amphiphilic polymer (RGDPEG-Chol) and develop an RGD-modified PTX-loaded liposome for targeted drug delivery. The RGD-modified PTXloaded liposome has been extended to evaluate physicochemical properties such as particle size, morphologies, and zeta potential. The drug release profile, cellular uptake, and antitumor activity of PTX liposomes were also investigated in vitro.

\section{Experimental Section}

2.1. Materials. Cholesterol (Chol) was obtained from BoAo Biological Technology (Shanghai, China). Monomethoxy poly(ethylene glycol) (MW 2000, mPEG2000), poly(ethylene glycol) (MW 2000, PEG2000), 3-[4,5-dimethylthiazol-2yl]-2,5-diphenyltetrazolium bromide (MTT), 1-(3-dimethylaminopropyl)-3-ethylcarbodiimide hydrochloride (EDCI), and 4-dimethylaminopyridine (DMAP) were purchased from Sigma-Aldrich (USA). 1,8-Diazabicyclo [5.4.0] undec-7ene (DBU), coumarin-6, fmoc-L-phenylalanine (fmoc-phe), and succinic anhydride (suc) were provided by Energy Chemical (Shanghai, China). 3-Maleimidopropionic acid Nsuccinimidyl ester (BMPS) was purchased from Chengdu
Boke Co. Ltd. (Chengdu, China). Soya phosphatidylcholine (SPC) was purchased from Lucas Meyer (Hamburg, Germany). Paclitaxel (PTX) was purchased from Yihe Biological Technology (Shanghai, China). Cysteine-glycinearginine-glycine-asparagine-serine (CGRGDS) hexapeptide was obtained from Chengdu Kaijie Co. Ltd. (Chengdu, China). All other solvents and reagents were of chemical grade and used without other purification. Ultrapure water from Milli-Q water system was used to prepare the aqueous solutions.

\subsection{Synthesis of Methoxy Poly(ethylene glycol)-Cholesterol} Conjugate. Methoxy poly(ethylene glycol)-cholesterol conjugate (mPEG-Chol) was synthesized according to literature [28-30] with minor modifications. As is displayed in Scheme 1, firstly, a solution of dichloromethane $(100 \mathrm{~mL})$ containing cholesterol $(5.0 \mathrm{~g}, 12.9 \mathrm{mmol})$, DMAP $(1.6 \mathrm{~g}$, $12.9 \mathrm{mmol})$, and succinic anhydride $(6.5 \mathrm{~g}, 66.7 \mathrm{mmol})$ was stirred at room temperature for $48 \mathrm{~h}$. Thereafter most dichloromethane was removed using rotary evaporator and the crude solution was poured into acetic acid $(150 \mathrm{~mL})$. The precipitates were filtered and washed three times with acetic acid, and cholesterol monosuccinate (Chol-suc) (5.8 g $92.5 \%)$ was finally acquired by vacuum drying overnight. Secondly, MPEG (2.0 g, $1.0 \mathrm{mmol})$, Chol-suc $(0.72 \mathrm{~g}, 1.5 \mathrm{mmol})$, DMAP $(0.13 \mathrm{~g}, 1.0 \mathrm{mmol})$, and EDCI $(0.48 \mathrm{~g}, 2.5 \mathrm{mmol})$ were dissolved in dichloromethane $(100 \mathrm{~mL})$ and refluxed at room temperature for $24 \mathrm{~h}$. The reaction mixture was then washed with $1 \mathrm{~N} \mathrm{HCl}$ solution, saturated in $\mathrm{NaCl}$ solution, and dried over anhydrous $\mathrm{Na}_{2} \mathrm{SO}_{4}$. The solvent was evaporated and the residue was purified with column chromatography (methanol: dichloromethane $=1: 20$ ) to give mPEG-Chol (2.12 g, 86.1\%).

2.3. Synthesis of Cholesterol-Poly(ethylene glycol)-RGD Peptides Conjugate. The synthesis process of cholesterol-poly(ethyleneglycol)-RGD conjugate (RGD-PEG-Chol) was shown in Scheme 2.

2.3.1. Synthesis of Poly(ethylene glycol)-Phe-Fmoc Conjugate (PEG-Phe-Fmoc). A dichloromethane solution $(150 \mathrm{~mL})$ of fmoc-phe $(1.45 \mathrm{~g}, 3.75 \mathrm{mmol})$, DMAP $(0.31 \mathrm{~g}, 2.5 \mathrm{mmol})$, EDCI $(0.72 \mathrm{~g}, 3.75 \mathrm{mmol})$, and PEG $(5.0 \mathrm{~g}, 2.5 \mathrm{mmol})$ was stirred at room temperature for $48 \mathrm{~h}$. After completion of the reaction, the solution was washed with $1 \mathrm{~N} \mathrm{HCl}$ solution, saturated in $\mathrm{NaCl}$ solution, and then dried over anhydrous $\mathrm{Na}_{2} \mathrm{SO}_{4}$. The solvent was evaporated and the residue was purified with column chromatography (methanol: dichloromethane $=1: 60)$ to give the monofunctional product, poly(ethylene glycol)-phe-fmoc conjugate (PEG-Phe-Fmoc) (5.2 g, 87.8\%).

2.3.2. Synthesis of Cholesterol-Poly(ethylene glycol)-Phe- $\mathrm{NH}_{2}$ Conjugate (Chol-PEG-Phe- $\mathrm{NH}_{2}$ ). The mixture of PEG-PheFmoc $(5.0 \mathrm{~g}, 2.1 \mathrm{mmol})$, Chol-suc $(1.2 \mathrm{~g}, 2.5 \mathrm{mmol})$, DMAP $(0.25 \mathrm{~g}, 2.1 \mathrm{mmol})$, and EDCI $(0.8 \mathrm{~g}, 4.2 \mathrm{mmol})$ in dichloromethane $(150 \mathrm{~mL})$ was stirred at room temperature for $24 \mathrm{~h}$. After completion of the reaction, the solution was washed with $1 \mathrm{NHCl}$ solution, saturated $\mathrm{NaCl}$ solution, 


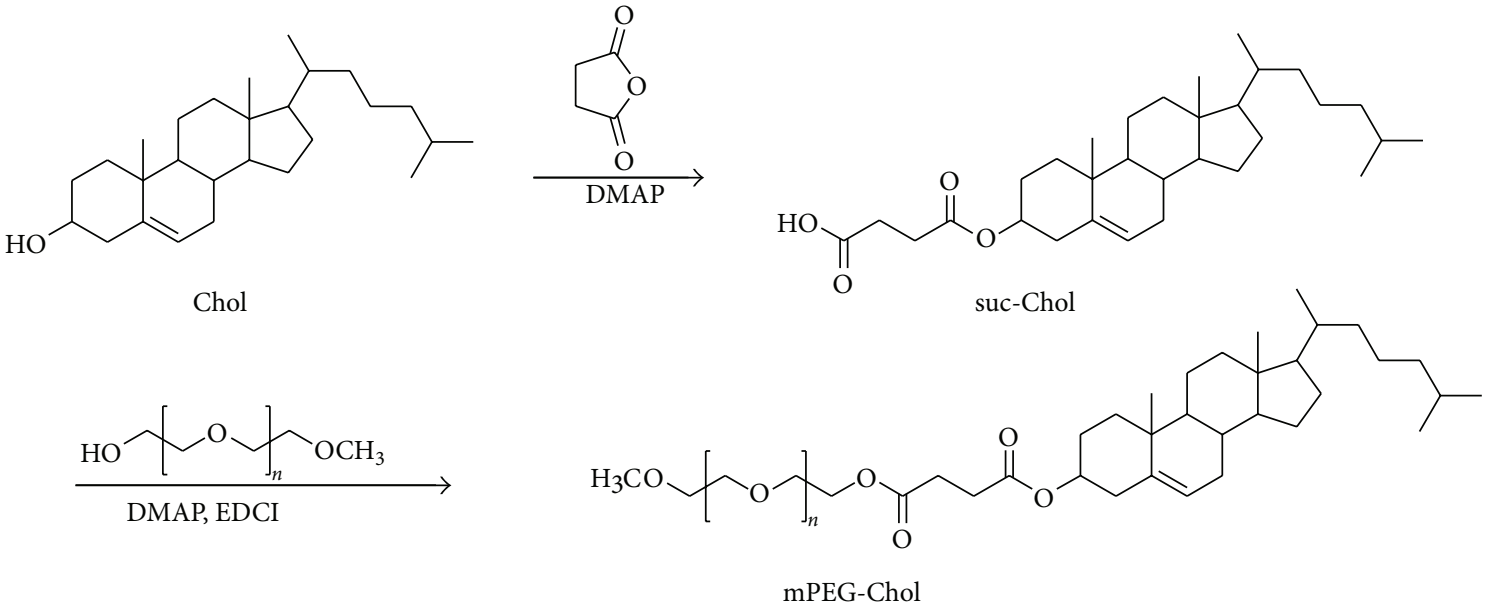

Scheme 1: Synthetic route of mPEG-Chol polymer.

and dried over anhydrous $\mathrm{Na}_{2} \mathrm{SO}_{4}$. The solution was concentrated under vacuum and cholesterol-poly(ethylene glycol)-phe-fmoc conjugate (Chol-PEG-Phe-Fmoc) $(5.2 \mathrm{~g}$, $87.2 \%$ ) was obtained after being purified on a silicagel column chromatography (methanol : dichloromethane $=$ $1: 40)$. Chol-PEG-Phe-Fmoc $(3.0 \mathrm{~g}, 1.1 \mathrm{mmol})$ dissolved in dichloromethane $(50 \mathrm{~mL})$ was refluxed at room temperature for $10 \mathrm{~min}$; then $\mathrm{DBU}(0.19 \mathrm{~g}, 1.3 \mathrm{mmol})$ was added. After being refluxed at room temperature for $40 \mathrm{~min}$, the solution was washed directly with $1 \mathrm{~N} \mathrm{HCl}$ solution, saturated in $\mathrm{NaCl}$ solution, and dried over anhydrous $\mathrm{Na}_{2} \mathrm{SO}_{4}$. Dichloromethane was removed using rotary evaporator and the crude solution was poured into ethyl ether $(100 \mathrm{~mL})$. The precipitated solid was separated by filtration, washed three times with ethyl ether, finally, the Chol-PEG-Phe- $\mathrm{NH}_{2}(2.4 \mathrm{~g}$, $83.6 \%$ ) was obtained by vacuum drying overnight.

2.3.3. Synthesis of RGD-PEG-Chol. A mixture of Chol-PEGPhe- $\mathrm{NH}_{2}(2.0 \mathrm{~g}, 0.76 \mathrm{mmol})$ and BMPS $(0.24 \mathrm{~g}, 0.92 \mathrm{mmol})$ in DMSO $(50 \mathrm{~mL})$ was stirred at room temperature for $24 \mathrm{~h}$ to give a 3-maleimidopropionic acid linker on PEG chain. The peptide CGRGDS $(0.55 \mathrm{~g}, 0.92 \mathrm{mmol})$ was added and stirred for another $24 \mathrm{~h}$. After completion of the reaction, the solution was dialyzed successively against $\mathrm{H}_{2} \mathrm{O}$ (membrane tubing, molecular weight cutoff $1000 \mathrm{Da}$ ). The product after dialysis was then lyophilized and RGD-PEG-Chol (1.2 g, $47.1 \%)$ was obtained.

2.4. Cell Cultures. The murine B16F10 cell lines were obtained from the Basic Medical Cell Center, Chinese Academy of Medical Science (CAMS, Beijing, China) and were cultured in DMEM medium (Gibco, Paisley, UK) supplemented with 100 units/mL penicillin, 100 units/mL streptomycin, and $10 \%$ heat-inactive fetal calf serum and incubated in a humidified atmosphere containing $5 \% \mathrm{CO}_{2}$ at $37^{\circ} \mathrm{C}$. Human umbilical vein endothelial cells (HUVECs) were isolated from umbilical cords, cultured in EGM-2 medium supplemented with 100 units/mL penicillin and 100 units/mL streptomycin.
The concentration of cells was determined by counting trypsinized cells with a hemocytometer.

2.5. Liposomes Preparation and Drug Loading. Conventional liposomes (CL) were prepared by a thin film dispersion method. Accurately weighed SPC (39 mg), Chol (13 mg), mPEG-Chol (10 mg), and PTX (0.667 mg) were first dissolved in $4 \mathrm{~mL}$ chloroform in a round-bottom flask, dried into transparent thin film on a rotary evaporator under vacuum at $30^{\circ} \mathrm{C}$, and followed by vacuum dry overnight to remove any traces of organic solvent. The lipid film was hydrated by $8 \mathrm{~mL}$ of Milli-Q water at $60^{\circ} \mathrm{C}$ for $20 \mathrm{~min}$, after which the suspension was dispersed by a probe-type ultrasonicator for $5 \mathrm{~min}$ and sequentially extruded through a $0.8 \mu \mathrm{m}$ and $0.2 \mu \mathrm{m}$ pore size polycarbonate filter (Nuclepore, USA).

RGD-modified liposomes (CGRGDS) were obtained by the same method above, except that mPEG-Chol and RGD-PEG-Chol polymers with different mass ratios (from $10 \mathrm{mg} / 0 \mathrm{mg}$ to $0 \mathrm{mg} / 10 \mathrm{mg}$ ) were dissolved in chloroform along with SPC, Chol and PTX, or coumarin- 6 before the thin film was prepared.

To determine the drug loading (DL) and encapsulation efficiency (EE) of the PTX-loaded liposomes, $1 \mathrm{~mL}$ liposome was diluted in $1 \mathrm{~mL}$ methanol, and then the PTX content was determined by high-performance liquid chromatography (HPLC). In the analysis, a LC-20AD (Shimadzu Corp., Japan) apparatus equipped with a SPD-20A UV detector (Shimadzu Corp., Japan) and a DIONEX Acclaim 120 RP-C18 column (DIONEX Corp., USA) $(250 \times 4.6 \mathrm{~mm})$ were used. The mobile phase was composed of $60: 40(\mathrm{v} / \mathrm{v})$ acetonitrile-water with a flow rate of $1 \mathrm{~mL} / \mathrm{min}$ and the detection wavelength was set at $227 \mathrm{~nm}$. The DL and EE of the liposomes were calculated using the following formulas:

$$
\begin{gathered}
\mathrm{DL}=\frac{\text { weight of drug in liposome }}{\text { weight of feeding polymer and drug }} \times 100 \%, \\
\mathrm{EE}=\frac{\text { weight of drug in liposome }}{\text { weight of drug feed }} \times 100 \% .
\end{gathered}
$$




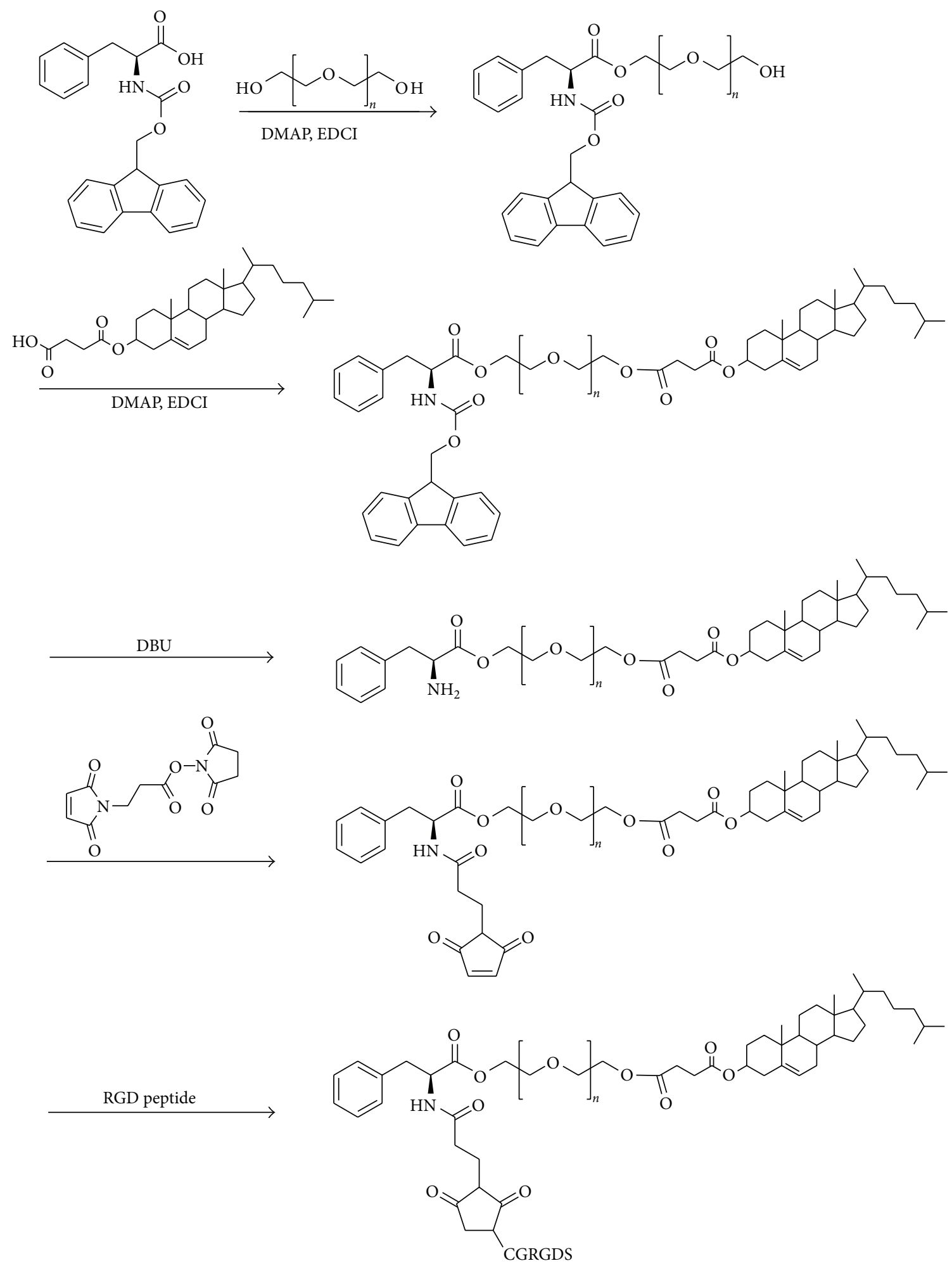

SCHEME 2: Synthetic route of RGD-PEG-Chol polymer.

2.6. Size Determination and Zeta Potential. Particle size of the liposome was determined by Dynamic light scattering (DLS) with a Zetasizer Nano ZS-90 instrument (Malvern Instruments, Malvern, UK). Zeta potential was monitored using the same instrument as size measurement. Zeta limits ranged from -150 to $150 \mathrm{mV}$. The liposome suspension was diluted 10 times before measurement. During the progress, refractive index was 1.5000 , and temperature was kept at $25^{\circ} \mathrm{C}$. 
Each test was measured for 3 times and mean value was taken.

2.7. Transmit Electronic Microscopy (TEM). A transmission electron microscope (TEM) was used to observe the morphology of prepared liposome. Liposome was diluted 10 times with distilled water and placed on a copper grid covered with nitrocellulose. The sample was negatively stained with phosphotungstic acid and dried at room temperature, after which TEM images were taken by a transmission electron microscope (H-6009IV, Hitachi, Japan).

2.8. In Vitro Drug Release. The release profiles of PTX from RGD-modified liposome and free PTX were investigated by the dialysis method. $1 \mathrm{~mL}$ drug loaded liposome or free PTX was placed into dialysis bags (molecular weight cutoff $=3500$ ) which were then incubated in $50 \mathrm{~mL}$ phosphate buffered solution (PBS) (PH 7.4 or PH 5.5, $0.01 \mathrm{M}$ ) containing Tween $80(0.5 \% \mathrm{wt})$ at $37^{\circ} \mathrm{C}$ with gentle shaking $(100 \mathrm{rpm})$. At given time points, $1 \mathrm{~mL}$ dialysis medium was withdrawn and replaced with same volume of fresh buffer. To calculate cumulative amount of released PTX, the samples were analyzed by HPLC. All the results were the mean value of three test runs and all data were shown as the mean \pm SD.

2.9. Cellular Uptake of Liposomes by Flow Cytometry Analysis. An aliquot of $1.5 \mathrm{~mL}$ of HUVEC cells suspension $(6 \times$ $10^{4}$ cells/well) was seeded in a six-well tissue culture plate (Corning, NY, USA) and was incubated for $24 \mathrm{~h}$ at $37^{\circ} \mathrm{C}$. Then $30 \mu \mathrm{L}$ coumarin- 6 loaded conventional liposomes or RGDmodified liposomes were added to each well, respectively, and the final concentration of coumarin-6 is $40 \mathrm{ng} / \mathrm{mL}$. The plates were incubated at $37^{\circ} \mathrm{C}$ for another $1 \mathrm{~h}$, after which the medium was discarded and cell monolayer was suspended by treatment with trypsin and washed three times with cold PBS. Then the cell samples were examined by a flow cytometer (EPICS Elite ESP, Beckman Coulter, USA). The intracellular coumarin-6 was excited with an argon laser $(488 \mathrm{~nm})$, and fluorescence was detected at $525 \mathrm{~nm}$. Files were collected from 10,000 gated events.

2.10. In Vitro Cytotoxicity Assay. A comparison of in vitro cytotoxicity of various PTX formulations was performed on B16F10 cells with an in vitro proliferation using MTT method. Briefly, 3000 cells in $100 \mu \mathrm{l}$ DMEM were plated in 96-well plates and incubated for $24 \mathrm{~h}$ at $37^{\circ} \mathrm{C}$ in humanized atmosphere containing $5 \% \mathrm{CO}_{2}$. These cells were exposed to different concentrations of free PTX, blank liposome, PTXloaded liposome (mPEG/PTX Lipo), and RGD-modified PTX-loaded liposome (RGD/PTX Lipo) at $37^{\circ} \mathrm{C}$ for $48 \mathrm{~h}$. $20 \mu \mathrm{l}$ of MTT solution $(5 \mathrm{mg} / \mathrm{mL}$ dissolved in physiological saline) was added and incubated for another $4 \mathrm{~h}$, and then the media were replaced with $150 \mu \mathrm{l}$ DMSO. After slight shake for $10 \mathrm{~min}$, the absorbance was read on a Spectramax M5 Microtiter Plate Luminometer (Molecular Devices, US) at dual wavelength of $490 \mathrm{~nm}$. Each experiment was repeated three times in triplicate $(n=9)$. The data reports represented the means of triplicate measurement.

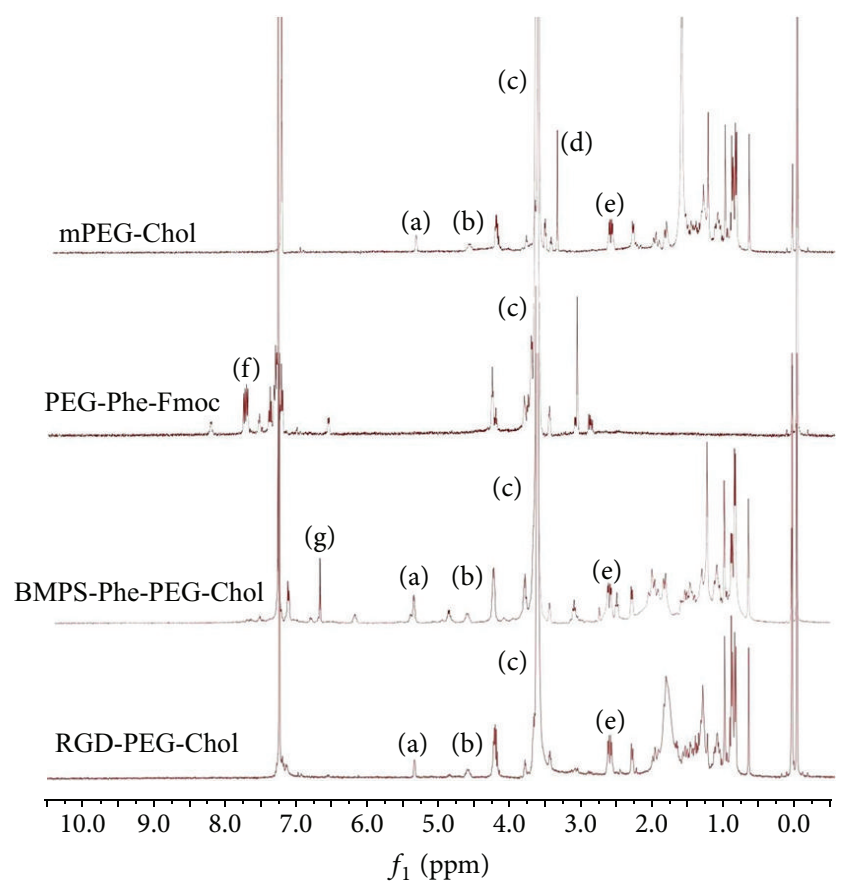

Figure 1: ${ }^{1} \mathrm{H}-\mathrm{NMR}$ spectra of mPEG-PEG, PEG-Phe-Fmoc, BMPSPhe-PEG-Chol, and RGD-PEG-Chol in $\mathrm{CDCl}_{3}$. (a, b) 6- and 3position protons in cholesterol; (c) the repeating units of methoxyl in $\mathrm{mPEG}$ or PEG; (d) the protons in the terminal group of mPEG; (e) methylene proton of succinyl group; (f) protons in the Fmoc group; (g) protons on maleimidyl group of linkers.

2.11. Statistics Analysis. All data were reported as means \pm standard deviation (SD). Statistical significance between pairs of samples was measured using 2-tailed Student's $t$-test. A value of $P<0.05$ was considered statistically significant.

\section{Results and Discussion}

3.1. Synthesis of RGD-PEG-Chol. As shown in Figure 1, the multiplet at $\delta 3.5-3.8$ was attributed to the repeating units $\left(-\mathrm{CH}_{2}, \mathrm{c}\right)$ in PEG or mPEG. And the multiple signals at $\delta$ 7.40-7.78 came from benzene ring from Fmoc. The multiple signals at $\delta 0.67-2.20$ came from the protons in cholesterol. The six-position protons $(-\mathrm{CH}=\mathrm{CH}-$, a $)$ in cholesterol were found at $\delta$ 5.36. Signals at $\delta 7.12-7.36$ were assigned to phenylalanine. As shown in the spectra of Linker-PEG-Chol, cholesterol and 3-maleimidopropionic acid linker had been connected to PEG chain successfully, and the peaks at $\delta$ 6.69 were attributed to the protons of linker $(-\mathrm{CH}=\mathrm{CH}-, \mathrm{h})$. This result demonstrated that the linker had been conjugated to the Chol-PEG. The weak peaks at $\delta 4.0-4.2$ belonged to protons of RGD $\left(-\mathrm{NHCH}\left(\mathrm{CH}_{2}\right) \mathrm{CO}-\right.$, e) while peaks at $\delta 6.69$ disappeared which indicated that the $-\mathrm{CH}=\mathrm{CH}-$ had been broken. The whole results told us that the synthesis of RGDPEG-Chol was successful.

The FT-IR spectra of Fmoc-phe-PEG, Fmoc-phe-PEGChol, and Chol-PEG -RGD were shown in Figure 2. The broad band at around $3440 \mathrm{~cm}^{-1}$ was attributed to the $-\mathrm{NH}_{2}$ stretching vibration of phenylalanine. The sharp peak 


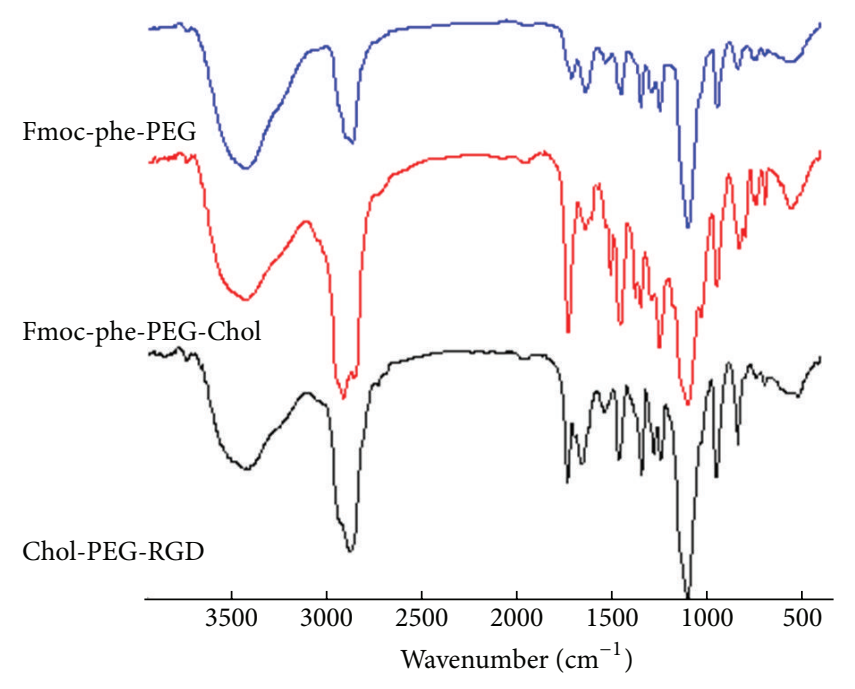

FIgUre 2: The FT-IR spectra of Fmoc-phe-PEG, Fmoc-phe-PEGChol, and RGD-PEG-Chol.

at $1733 \mathrm{~cm}^{-1}$ was assigned to the $-\mathrm{C}=\mathrm{O}$ of $-\mathrm{COOCO}-$ group which was brought by the cholesterol monosuccinate. The peak at $2880 \mathrm{~cm}^{-1}$ was assigned to the $-\mathrm{CH}_{2} \mathrm{CH}_{2} \mathrm{O}$ which was brought by PEG. Other prominent peaks at $1282 \mathrm{~cm}^{-1}$ and $1246 \mathrm{~cm}^{-1}$ were assigned to the asymmetrical and symmetrical bending vibrations of $-\mathrm{C}-\mathrm{S}$ - which were the introduction of RGD chain after combination of $-\mathrm{SH}$ from cysteine to the linker.

3.2. Screening of Blank Liposome. The base lipid used in this experiment is cholesterol $13 \mathrm{mg}$, soy lecithin $39 \mathrm{mg}$, and the added polymers A: mPEG-Chol and B: RGD-PEG-Chol. The weight of A plus $B$ is kept at $10 \mathrm{mg}$, and different $A / B$ mass ratios were tested in the preparation process of liposomes. The results (Figure 3 ) show that as B RGD-PEG-Chol mass ratio increases, the particle size decreased from about $100 \mathrm{~nm}$ to $65 \mathrm{~nm}$ and that when mass ratios of $\mathrm{A} / \mathrm{B}=2: 8$ and $1: 9$, it achieved a smaller particle size, but when there was no polymer A, the particle size increased again. PDI and zeta potential of liposomes showed no obvious trend when the mass ratio of $\mathrm{A} / \mathrm{B}$ changes from $10: 0$ to $0: 10$.

3.3. The Drug Loading and Final Screening of A/B Mass Ratio. Based on the results of previous section, we selected $A / B$ mass ratios of $2 / 8$ and $1 / 9$, which bring optimum particle size and PDI results. Paclitaxel was employed in liposome formulation. In this part, we set different mass ratios of $(\mathrm{A}+$ B)/PTX as 10:1, 15:1, 20:1, and 30:1 to prepare liposomes encapsulating various amount of PTX. Then their size, PDI, zeta potential, drug loading (DL\%), and the encapsulation efficiency (EE\%) were determined. It is confirmed (Tables 1 and 2) that, when $A / B$ mass ratio was set as $1: 9$, the parameters were better than those with $A / B$ mass ratio of $2: 8$, and when we set mass ratio of $(\mathrm{A}+\mathrm{B}) / \mathrm{PTX}$ as $15: 1$, after comprehensive assessment of particle size, PDI, EE\%, and DL\%, we got optimal PTX-liposome, which has a size of

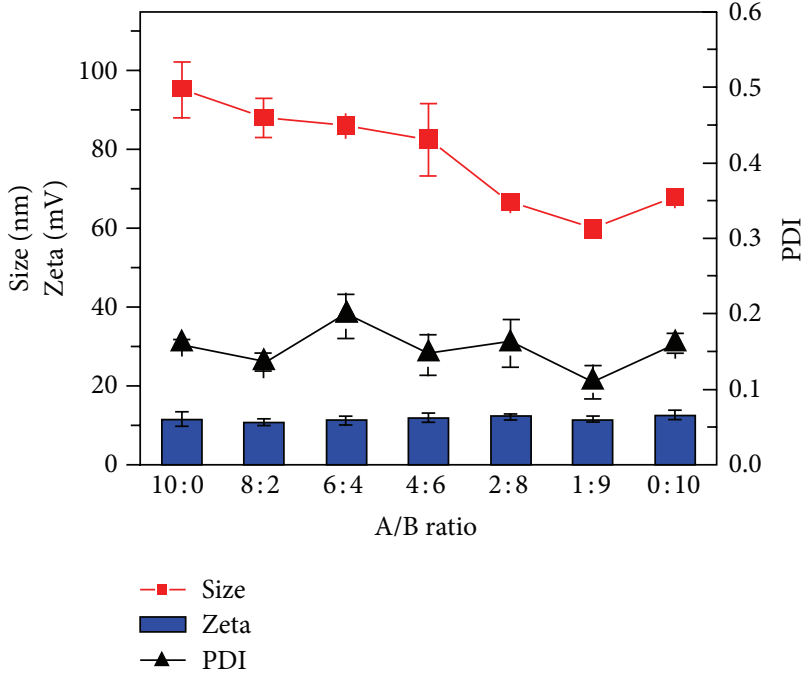

FIGURE 3: Screening of blank liposome.

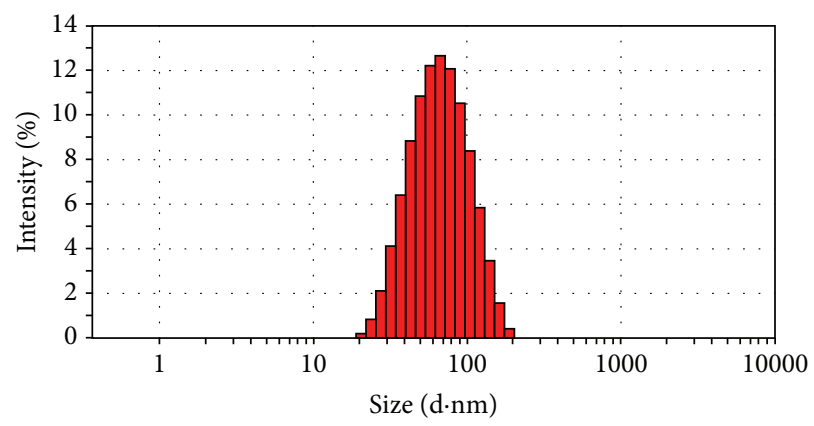

FIGURE 4: Size distribution of RGD-PTX liposomes.

$58.94 \mathrm{~nm}$, a PDI of 0.154 , zeta potential of $16.3 \mathrm{mv}, \mathrm{DL} \%$ of $1.15 \%$, and $\mathrm{EE} \%$ of $99.12 \%$.

3.4. Characterization of RGD-Modified PTX-Loaded Liposome. The mean size of RGD-modified PTX-loaded liposome (RGD-PTX liposome) was $58.94 \pm 4.82$ (mean \pm SD; $n=3$ ) as shown in Figure 4, polydispersity index of $0.154 \pm$ 0.10 (mean $\pm \mathrm{SD} ; n=3$ ), with zeta potential of $16.3 \pm 0.1 \mathrm{mv}$ (mean $\pm S D ; n=3$ ) which was shown in Figure 5 . When those preparations were stored at $4^{\circ} \mathrm{C}$, there is no significant change of size within 28 days (data not shown). Further transmit electronic microscopy analyses confirmed that the liposomes were kept in a good form with suitable size which was shown in Figure 6. The HPLC results showed that the load dose of RGD-PTX liposome was $>1 \%$ and the entrapment efficiency was $>99 \%$.

Drug-containing liposomes which have diameters with ranges of approximately $60-150 \mathrm{~nm}$ are small enough to extravasate from the blood vessels into the tumor interstitial space through their pores. In contrast, the blood vessels in most normal tissues are nonfenestrated capillaries and liposome with $\sim 120 \mathrm{~nm}$ in mean diameter did not accumulate in these normal tissues after i.v. injection. The particle size of 
TABLE 1: Data of PTX liposome when mass ratio of A/B is $1: 9$.

\begin{tabular}{|c|c|c|c|c|c|}
\hline$(\mathrm{A}+\mathrm{B}) / \mathrm{PTX}$ & Size/nm & PDI & Zeta/mv & $\mathrm{DL} / \%$ & $\mathrm{EE} \%$ \\
\hline $10: 1$ & $110.3 \pm 5.15$ & $0.198 \pm 0.31$ & $20.1 \pm 0.3$ & $1.62 \pm 0.021$ & $97.25 \pm 0.13$ \\
\hline $15: 1$ & $58.94 \pm 4.82$ & $0.154 \pm 0.10$ & $16.3 \pm 0.1$ & $1.15 \pm 0.016$ & $99.12 \pm 0.07$ \\
\hline $20: 1$ & $62.49 \pm 3.77$ & $0.130 \pm 0.21$ & $14.7 \pm 0.5$ & $0.08 \pm 0.008$ & $99.56 \pm 0.02$ \\
\hline $30: 1$ & $61.18 \pm 2.15$ & $0.113 \pm 0.17$ & $14.4 \pm 0.7$ & $0.05 \pm 0.005$ & $99.79 \pm 0.02$ \\
\hline
\end{tabular}

Note: A: mPEG-Chol B: RGD-PEG-Chol A/B = 1: 9.

TABLE 2: Data of PTX liposome when mass ratio of A/B is $2: 8$.

\begin{tabular}{|c|c|c|c|c|c|}
\hline$(\mathrm{A}+\mathrm{B}) / \mathrm{PTX}$ & Size/nm & PDI & Zeta/mV & $\mathrm{DL} / \%$ & $\mathrm{EE} \%$ \\
\hline $10: 1$ & $111.9 \pm 7.8$ & $0.175 \pm 0.5$ & $26.5 \pm 0.3$ & $1.48 \pm 0.017$ & $95.11 \pm 0.24$ \\
\hline $15: 1$ & $71.36 \pm 6.1$ & $0.192 \pm 0.17$ & $19.1 \pm 0.7$ & $1.06 \pm 0.022$ & $98.85 \pm 0.33$ \\
\hline $20: 1$ & $63.76 \pm 5.2$ & $0.172 \pm 0.32$ & $16.7 \pm 1.1$ & $0.81 \pm 0.015$ & $99.12 \pm 0.25$ \\
\hline $30: 1$ & $66.11 \pm 4.7$ & $0.144 \pm 0.11$ & $13.2 \pm 0.8$ & $0.53 \pm 0.012$ & $99.7 \pm 0.19$ \\
\hline
\end{tabular}

Note: A: mPEG-Chol B: RGD-PEG-Chol A/B = 2:8.

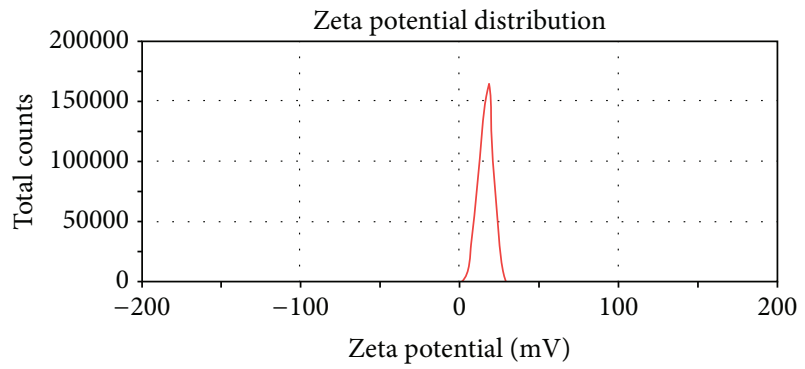

FIGURE 5: Zeta of RGD-PTX liposomes.

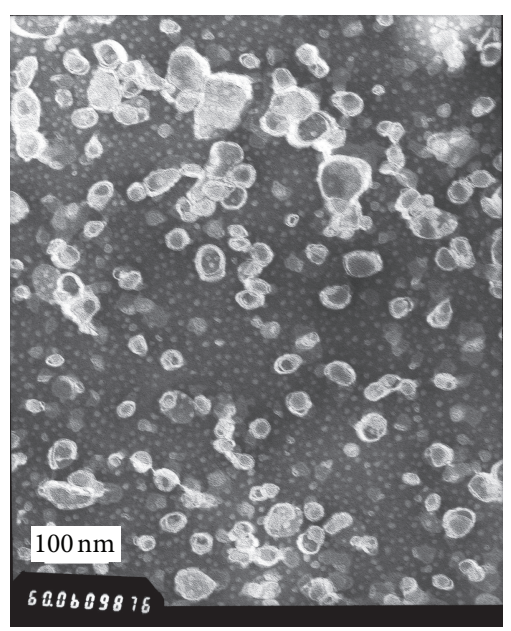

Figure 6: Typical TEM images of RGD-PTX liposomes.

RGD-PTX liposome was below $100 \mathrm{~nm}$ with narrow distribution and high entrapment efficiency [31]. And this encourages us to hypothesize it may be possible that the liposome we made could obtain an improved therapeutic efficacy since it may accumulate more readily in tumor tissues.

3.5. Stability Test for RGD/PTX Liposomes. Liposomes were sealed at $4^{\circ} \mathrm{C}$ in refrigerator, and the associated data were

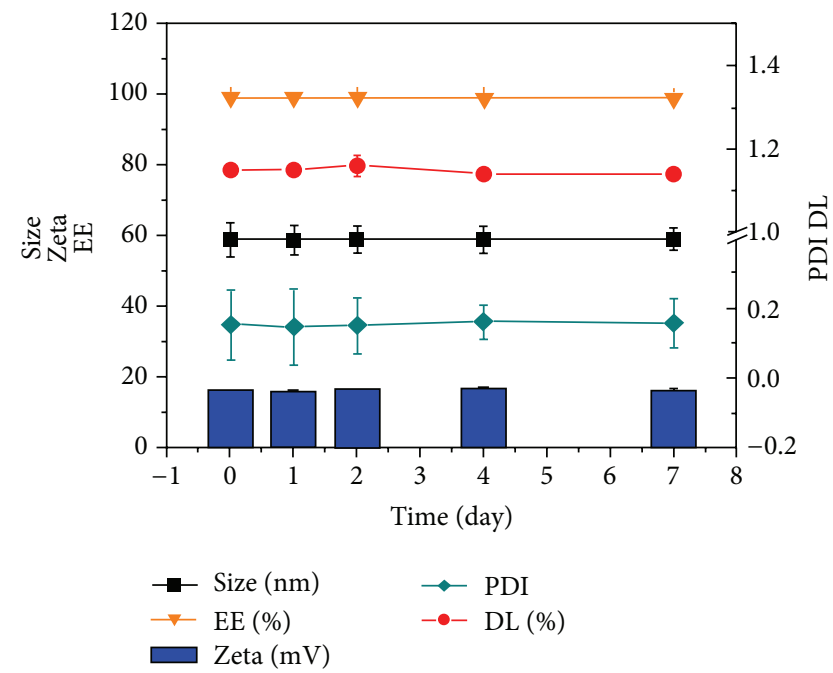

FIgURE 7: The stability of RGD/PTX liposomes in 7 days.

measured at the set time point; the results confirmed that the prepared RGD/PTX liposomes were stable and showed no significant change in seven days (shown in Figure 7).

3.6. In Vitro Release Behavior of RGD/PTX Liposome. Figure 8 shows the in vitro release profiles of free PTX and PTX-loaded RGD liposome in PBS at pH 5.5 and at $\mathrm{pH}$ 7.4. As expected, in $\mathrm{PBS}$ at $\mathrm{pH} 5.5$ and $\mathrm{pH} 7.4$ free PTX showed a very fast release profile, whereas RGD-PTX liposome released PTX in a much slower way in both release media. In the first 24 hours, approximately up to $90 \%$ of drug was released in free PTX. In comparison, within the same period only $40 \%$ of PTX in RGD liposome in PBS at $\mathrm{PH} 7.4$ was released, and less than $50 \%$ of PTX was released in PBS at $\mathrm{pH}$ 5.5. After 24 hours the release rate of PTX slowed down compared with the previous period of time and there was clearly a sustained release for at least 96 hours. The cumulative release rate of RGD-PTX liposome was $59.02 \pm$ 4.09 at $\mathrm{pH} 7.4$ and $65.32 \pm 3.88$ at $\mathrm{pH} 5.5$, much slower 


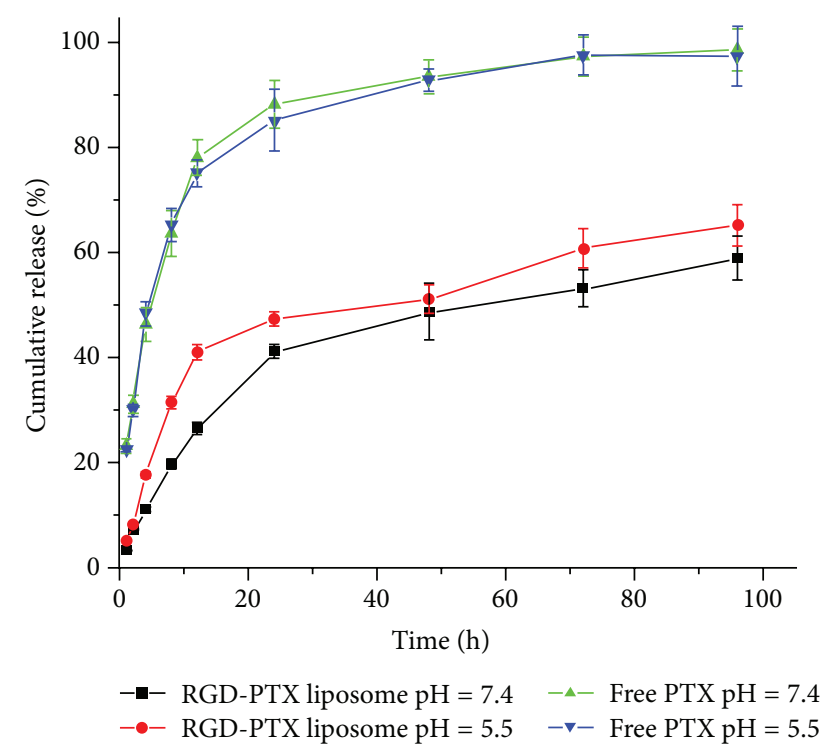

FIGURE 8: Drug release profiles of free PTX and RGD-PTX liposome in $\mathrm{PBS}$ solution at $\mathrm{pH} 7.4$ and $\mathrm{pH} 5.8$ with error bars showing the standard deviation $(n=3)$.

than that of free PTX $(98.67 \pm 4.02)$. RGD-PTX liposome released PTX slightly faster in PBS with lower $\mathrm{pH}$ which indicated that RGD-PTX liposome might be $\mathrm{pH}$-sensitive, which can be explained by faster hydrolysis of lipids at lower $\mathrm{pH}$. Also, this phenomenon can be attributed to the tendency that the oxygen atom on the hydrophilic end of RGD-PEGChol polymer would absorb more positive hydrogen ions and produce electrostatic repulsion which would destabilize liposome and increase drug release rate [32]. Because the microenvironments of intratumoral regions and intracellular compartments such as endosomes and lysosomes are acidic, RGD-PTX liposome might selectively deliver and release PTX to tumor tissue, indicating their potential applicability as a drug delivery system with reduced side effects to healthy tissues and increased drug concentration at the tumor site.

3.7. Cellular Uptake of Liposomes by Flow Cytometry Analysis. A flow cytometry analysis was used to determine the cellular uptake of mPEG/coumarin-6 liposome, RGD/coumarin-6 liposome, blank liposome (control). Figure 9 showed that uptake of RGD/coumarin-6 liposome by HUVECs was more than that of mPEG/coumarin-6 liposome after $1 \mathrm{~h}$ incubation. The mean fluorescence intensity of coumarin- 6 uptaken by HUVECs treated with RGD liposome was increased by 50 percent (mean $=15.87$ versus 23.53 arbitrary units) compared with that treated with mPEG liposome. These results confirmed that RGD modification enhanced uptake of liposomal drug by integrin-overexpressing HUVECs.

3.8. Cell Cytotoxicity of RGD-PTX Liposome. The cytotoxicity of RGD-PTX liposome, PTX liposome, blank liposome, and free PTX to B16F10 cells was compared. As shown in Figure 10, the $\mathrm{IC}_{50}$ value of RGD-PTX liposome was decreased to $0.079 \mu \mathrm{g} / \mathrm{mL}$ compared with $9.57 \mu \mathrm{g} / \mathrm{mL}$ of free

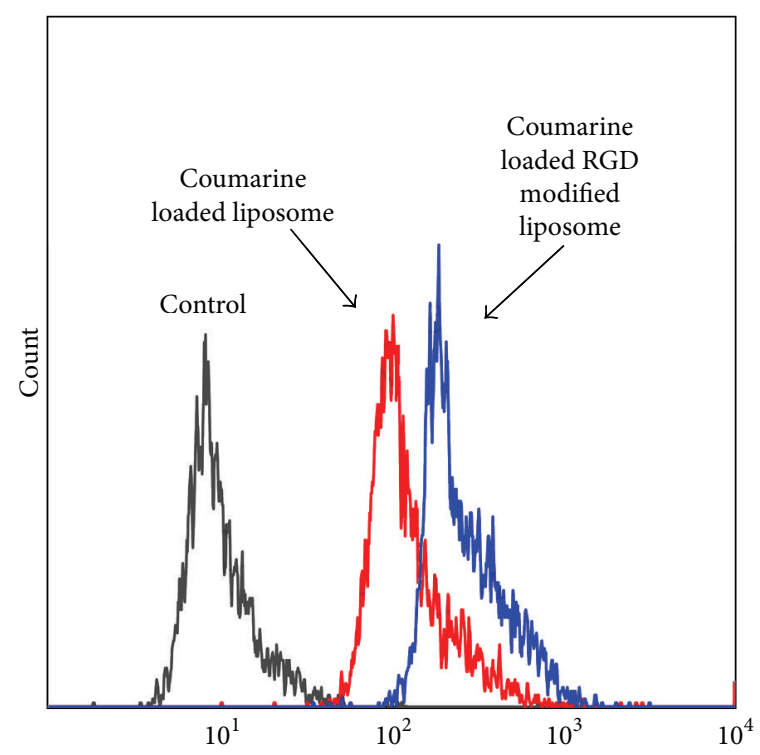

FIGURE 9: Flow cytometry profiles showed different cellular uptake of coumarin loaded liposomes with or without Chol-PEG-Chol by HUVEC cells (containing $40 \mathrm{ng} / \mathrm{mL}$ coumarin at $37^{\circ} \mathrm{C}$ for 1 hour).

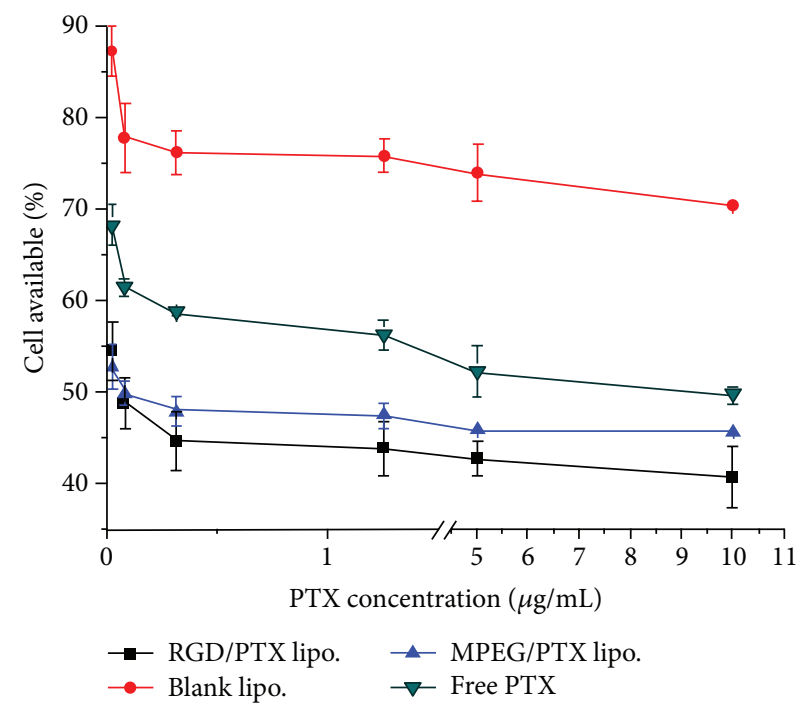

FIGURe 10: Cytotoxicity of free PTX, blank liposome, PTX liposome, and RGD-PTX liposome for B16F10 tumor cells.

PTX and $0.302 \mu \mathrm{g} / \mathrm{mL}$ of PTX liposome, respectively. The RGD-PTX liposome revealed more than 121-fold and 4fold increase of cytotoxicity in comparison to free PTX and PTX liposome. The blank liposomes show no apparent cytotoxicity to B16F10 cells. The results may be explained by two mechanisms: firstly, the RGD fragments chemically conjugated to the Chol-PEG polymer enabled the PTXloaded liposome to effectively target the surface of B16F10, since increased expression of $\alpha_{\mathrm{v}} \beta_{3}$ integrins was seen in melanoma cells and the RGD peptide can specifically bind to the $\alpha_{v} \beta_{3}$ integrins on melanoma tumor cells [33]; secondly, the RGD-PTX liposome drug delivery system increased 
solubility of poorly soluble drugs, which persistently released the drug and enhanced the effect of PTX.

\section{Conclusions}

In summary, a novel amphiphilic polymer Chol-PEG-RDG has been synthesized and characterized. The RGD/PTX liposomes have been successfully developed as a potential drug delivery system and the poorly soluble antitumor drug paclitaxel as model is loaded into the liposome. The liposome is spheroid with regular shape, a size within $100 \mathrm{~nm}$ and showed increased cellular uptake and sustained in vitro drug release behavior. Flow cytometry analysis revealed that the cellular uptake of coumarin encapsulated in the RGD-PEGChol modified liposome was increased for HUVEC cells. The in vitro cytotoxicity evaluated on the B16F10 cell lines by the MTT assay shows that RGD-PTX liposome has significantly enhanced cytotoxicity of paclitaxel for melanoma cells. Although further investigation on the amphiphilic polymer is required, the findings of our study represent an important step in advancing the use of RGD-PEG-Chol polymer modified liposome as a potential strategy to novel drug delivery system.

\section{Conflict of Interests}

The authors declare that there is no conflict of interests.

\section{Authors' Contribution}

Shi Zeng and Fengbo Wu contributed equally to this work.

\section{Acknowledgments}

The authors apologize to the many research groups whose work could not be cited here due to space limitations. They gratefully acknowledge the support by the National Natural Science Foundation of China (nos. 81001357, 81273471, and 81303208) and the Open Research Fund of State Key Laboratory Breeding Base of Systematic Research, Development and Utilization of Chinese Medicine.

\section{References}

[1] A. J. McHugh, "The role of polymer membrane formation in sustained release drug delivery systems," Journal of Controlled Release, vol. 109, no. 1-3, pp. 211-221, 2005.

[2] A. K. Bajpai, S. K. Shukla, S. Bhanu, and S. Kankane, "Responsive polymers in controlled drug delivery," Progress in Polymer Science, vol. 33, no. 11, pp. 1088-1118, 2008.

[3] S. Sershen and J. West, "Implantable, polymeric systems for modulated drug delivery," Advanced Drug Delivery Reviews, vol. 54, no. 9, pp. 1225-1235, 2002.

[4] A. J. Halliday, S. E. Moulton, G. G. Wallace, and M. J. Cook, "Novel methods of antiepileptic drug delivery-polymer-based implants," Advanced Drug Delivery Reviews, vol. 64, no. 10, pp. 953-964.

[5] A. N. Lukyanov and V. P. Torchilin, "Micelles from lipid derivatives of water-soluble polymers as delivery systems for poorly soluble drugs," Advanced Drug Delivery Reviews, vol. 56, no. 9, pp. 1273-1289, 2004.

[6] Y. Wang and S. M. Grayson, "Approaches for the preparation of non-linear amphiphilic polymers and their applications to drug delivery," Advanced Drug Delivery Reviews, vol. 64, no. 9, pp. 852-865, 2012.

[7] K. Rostamizadeh, M. Vahedpour, and S. Bozorgi, "Synthesis, characterization and evaluation of computationally designed nanoparticles of molecular imprinted polymers as drug delivery systems," International Journal of Pharmaceutics, vol. 424, no. 12, pp. 67-75, 2012.

[8] D.-B. Yang, J.-B. Zhu, Z.-J. Huang, H.-X. Ren, and Z.-J. Zheng, "Synthesis and application of poly(ethylene glycol)-cholesterol $\left(\right.$ Chol-PEG $\left.{ }^{m}\right)$ conjugates in physicochemical characterization of nonionic surfactant vesicles," Colloids and Surfaces B, vol. 63, no. 2, pp. 192-199, 2008.

[9] C. Carrion, J. C. Domingo, and M. A. De Madariaga, "Preparation of long-circulating immunoliposomes using PEG-cholesterol conjugates: effect of the spacer arm between PEG and cholesterol on liposomal characteristics," Chemistry and Physics of Lipids, vol. 113, no. 1-2, pp. 97-110, 2001.

[10] T. S. Levchenko, R. Rammohan, A. N. Lukyanov, K. R. Whiteman, and V. P. Torchilin, "Liposome clearance in mice: the effect of a separate and combined presence of surface charge and polymer coating," International Journal of Pharmaceutics, vol. 240, no. 1-2, pp. 95-102, 2002.

[11] S. Dadashzadeh, A. M. Vali, and M. Rezaie, "The effect of PEG coating on in vitro cytotoxicity and in vivo disposition of topotecan loaded liposomes in rats," International Journal of Pharmaceutics, vol. 353, no. 1-2, pp. 251-259, 2008.

[12] G. Lin, L. Cosimbescu, N. J. Karin, and B. J. Tarasevich, "Injectable and thermosensitive PLGA-g-PEG hydrogels containing hydroxyapatite: preparation, characterization and in vitro release behavior," Biomedical Materials, vol. 7, no. 2, Article ID 024107, 2012.

[13] D. Liu, T. Wang, X. X. Liu, and Z. Tong, "Accelerated cell sheet detachment by copolymerizing hydrophilic PEG side chains into PNIPAm nanocomposite hydrogels," Biomedical Materials, vol. 7 , no. 5 .

[14] O. Garbuzenko, Y. Barenholz, and A. Priev, "Effect of grafted PEG on liposome size and on compressibility and packing of lipid bilayer," Chemistry and Physics of Lipids, vol. 135, no. 2, pp. 117-129, 2005.

[15] Y.-Y. Wang, L.-X. Lü, Z.-Q. Feng, Z.-D. Xiao, and N.-P. Huang, "Cellular compatibility of RGD-modified chitosan nanofibers with aligned or random orientation," Biomedical Materials, vol. 5, no. 5, Article ID 054112, 2010.

[16] R. S. Bon and H. Waldmann, "Bioactivity-guided navigation of chemical space," Accounts of Chemical Research, vol. 43, no. 8, pp. 1103-1114, 2010.

[17] U. Hersel, C. Dahmen, and H. Kessler, "RGD modified polymers: biomaterials for stimulated cell adhesion and beyond," Biomaterials, vol. 24, no. 24, pp. 4385-4415, 2003.

[18] F. Pastorino, C. Brignole, D. Di Paolo et al., "Targeting liposomal chemotherapy via both tumor cell-specific and tumor vasculature-specific ligands potentiates therapeutic efficacy," Cancer Research, vol. 66, no. 20, pp. 10073-10082, 2006.

[19] E. Ruoslahti, "RGD and other recognition sequences for integrins," Annual Review of Cell and Developmental Biology, vol. 12 , pp. 697-715, 1996. 
[20] J. Spratlin and M. B. Sawyer, "Pharmacogenetics of paclitaxel metabolism," Critical Reviews in Oncology/Hematology, vol. 61, no. 3, pp. 222-229, 2007.

[21] A. Javeed, M. Ashraf, A. Riaz, A. Ghafoor, S. Afzal, and M. M. Mukhtar, "Paclitaxel and immune system," European Journal of Pharmaceutical Sciences, vol. 38, no. 4, pp. 283-290, 2009.

[22] B. Thompson, N. Mignet, H. Hofland et al., "Neutral postgrafted colloidal particles for gene delivery," Bioconjugate Chemistry, vol. 16, no. 3, pp. 608-614, 2005.

[23] J. Leblond, N. Mignet, L. Leseurre et al., "Design, synthesis, and evaluation of enhanced DNA binding new lipopolythioureas," Bioconjugate Chemistry, vol. 17, no. 5, pp. 1200-1208, 2006.

[24] X. B. Zhao, N. Muthusamy, J. C. Byrd, and R. J. Lee, "Cholesterol as a bilayer anchor for PEGylation and targeting ligand in folate-receptor-targeted liposomes," Journal of Pharmaceutical Sciences, vol. 96, no. 9, pp. 2424-2435, 2007.

[25] J.-P. Xu, J. Ji, and J.-C. Shen, "The effect of a cholesterol liquid crystalline structure on osteoblast cell behavior," Biomedical Materials, vol. 4, no. 2, Article ID 025010, 2009.

[26] Y. Qin, H. Chen, W. Yuan et al., "Liposome formulated with TAT-modified cholesterol for enhancing the brain delivery," International Journal of Pharmaceutics, vol. 419, no. 1-2, pp. 8595, 2011.

[27] L. Coderch, J. Fonollosa, M. De Pera, J. Estelrich, A. De La Maza, and J. L. Parra, "Influence of cholesterol on liposome fluidity by EPR. Relationship with percutaneous absorption," Journal of Controlled Release, vol. 68, no. 1, pp. 85-95, 2000.

[28] X. Qian, J. Lai, S. Zhan, J. Zhang, L. Wang, and K. Tu, "Novel mePEG-ehol and mePEG-st mixed micellar systems for drug delivery: preparation and characterization," Rare Metal Materials and Engineering, vol. 37, p. 655.

[29] F. Wu, T. Liu, C. Chen, C. Song, X. R. Zheng, and G. He, "Glycyrrhetinic acid-poly(ethylene glycol)-glycyrrhetinic acid tri-block conjugates based self-assembled micelles for hepatic targeted delivery of poorly water soluble drug," The Scientific World Journal, vol. 2013, Article ID 913654, 10 pages, 2013.

[30] T. Mahmood and N. Akhtar, "Stability of a cosmetic multiple emulsion loaded with green tea extract," The Scientific World Journal, vol. 2013, Article ID 153695, 7 pages, 2013.

[31] X.-B. Xiong, Y. Huang, W.-L. Lu et al., "Enhanced intracellular delivery and improved antitumor efficacy of doxorubicin by sterically stabilized liposomes modified with a synthetic RGD mimetic," Journal of Controlled Release, vol. 107, no. 2, pp. 262275, 2005.

[32] J. M. Li, Z. Y. He, S. Yu et al., "Micelles based on methoxy poly (ethylene glycol)-cholesterol conjugate for controlled and targeted drug delivery of a poorly water soluble drug," Journal of Biomedical Nanotechnology, vol. 8, pp. 1-9, 2012.

[33] W. Li, B. Su, S. Meng et al., "RGD-targeted paramagnetic liposomes for early detection of tumor: in vitro and in vivo studies," European Journal of Radiology, vol. 80, no. 2, pp. 598606, 2011. 

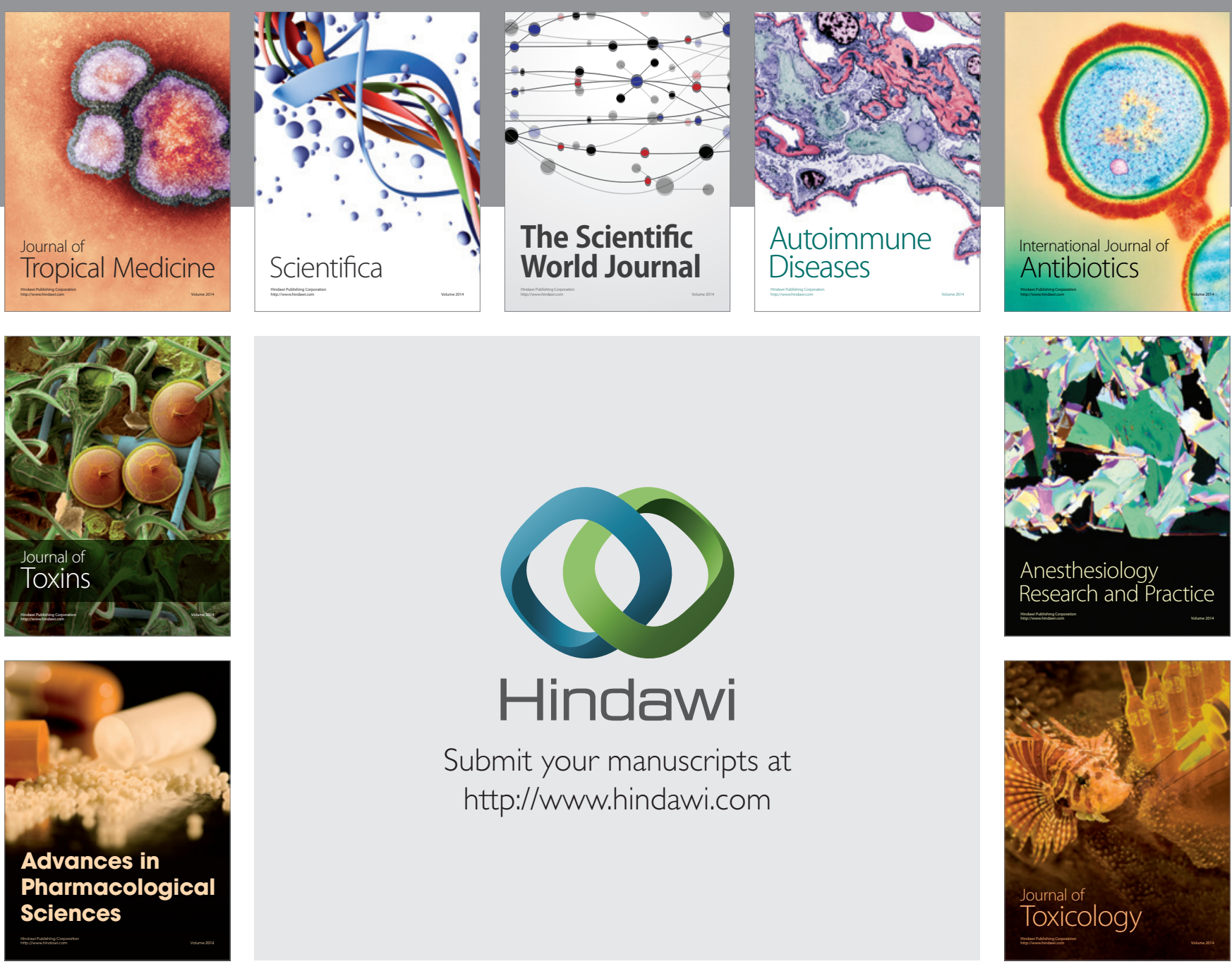

\section{Hindawi}

Submit your manuscripts at

http://www.hindawi.com
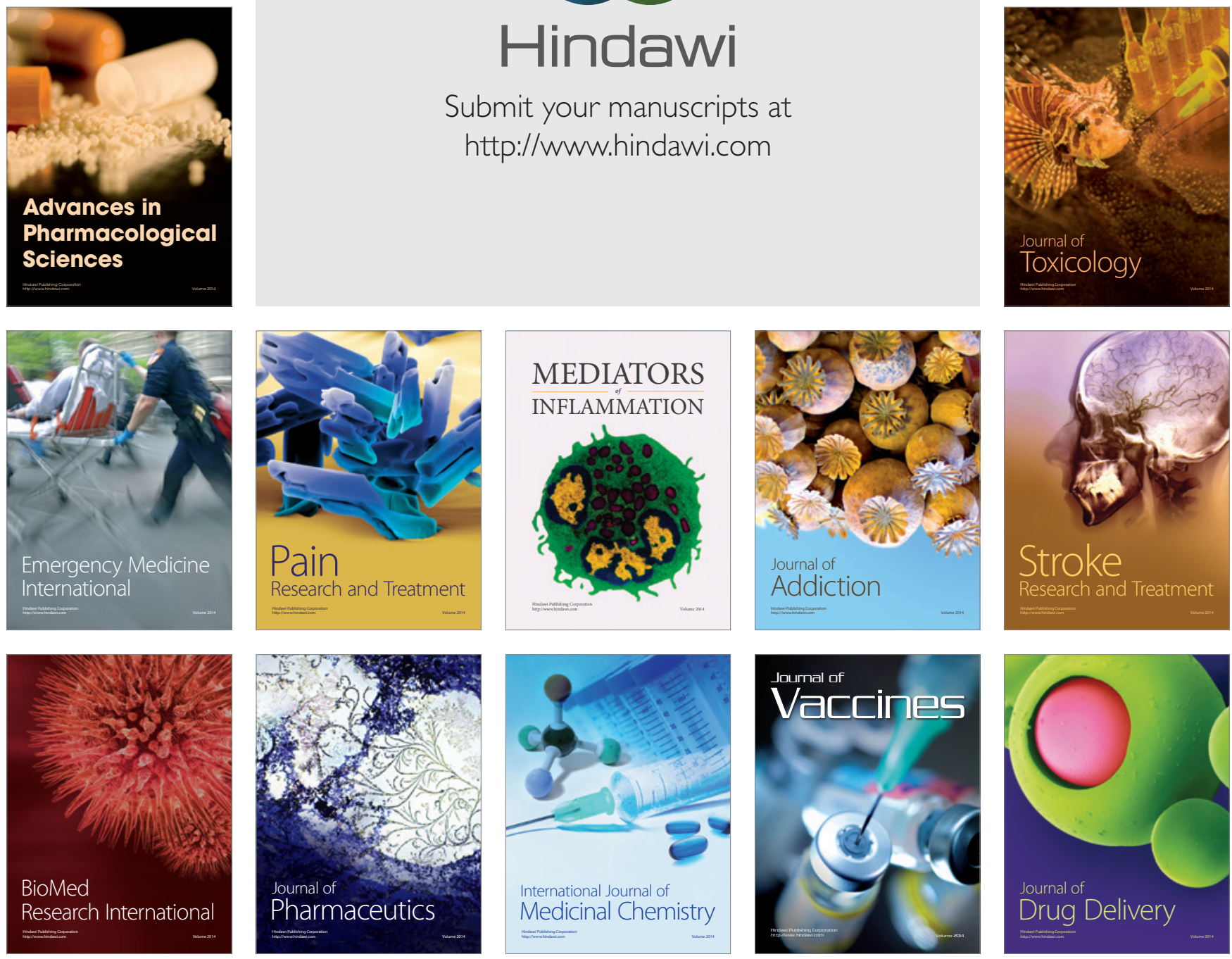